\title{
Hamiltonian Operator Approximation for Energy Measurement and Ground-State Preparation
}

\author{
Tatiana A. Bespalova $\odot^{1}$ and Oleksandr Kyriienko $\circledast^{2, *}$ \\ ${ }^{1}$ ITMO University, St. Petersburg, 197101, Russia \\ ${ }^{2}$ Department of Physics and Astronomy, University of Exeter, Stocker Road, Exeter EX4 4QL, United Kingdom
}

(Received 22 September 2020; revised 10 June 2021; accepted 7 July 2021; published 2 August 2021)

\begin{abstract}
The Hamiltonian operator plays a central role in quantum theory being a generator of unitary quantum dynamics. Its expectation value describes the energy of a quantum system. Typically being a nonunitary operator, the action of the Hamiltonian is either encoded using complex ancilla-based circuits, or implemented effectively as a sum of Pauli string terms. Here, we show how to approximate the Hamiltonian operator as a sum of propagators using a differential representation. The proposed approach, named the Hamiltonian operator approximation (HOA), is designed to benefit analog quantum simulators, where one has direct access to simulation of quantum dynamics, but measuring separate circuits is not possible. We describe how to use this strategy in the hybrid quantum-classical workflow for performing energy measurements. Benchmarking the measurement scheme, we discuss the relevance of the discretization step size, stencil order, number of shots, and noise. We also use HOA to prepare ground states of complex material science models with direct iteration and quantum filter diagonalization, finding the lowest energy for the 12-qubit Hamiltonian of hydrogen chain $\mathrm{H}_{6}$ with $10^{-5}$ Hartree precision using 11 time-evolved reference states. The approach is compared to the variational quantum eigensolver, proving that HOA is beneficial for systems at increasing size, corresponding to noisy large-scale quantum devices. We find that, for the Heisenberg model with 12 or more spins, our approach may outperform variational methods, both in terms of the gate depth and the total number of measurements.
\end{abstract}

DOI: 10.1103/PRXQuantum.2.030318

\section{INTRODUCTION}

Quantum computing is moving forward, and as evidenced by the quantum supremacy experiment [1], it is approaching the scale where high-performance classical computing may be challenged. However, despite everincreasing complexity for material science simulations [2-5], reaching quantum advantage requires both welltailored problems and modified quantum protocols to enable efficient computation.

While to date many quantum algorithms with favorable scaling have been proposed, they typically require deep gate sequences [6]. Considered suitable for the future fault-tolerant devices, their realization may be years (or even decades) away. Present day error-prone devices of increased size, corresponding to noisy intermediate-scale

\footnotetext{
*kyriienko@ukr.net

Published by the American Physical Society under the terms of the Creative Commons Attribution 4.0 International license. Further distribution of this work must maintain attribution to the author(s) and the published article's title, journal citation, and DOI.
}

quantum (NISQ) computers [7], require different strategies to solve a state preparation problem [8]. One solution corresponds to the adoption of a hybrid quantum-classical (HQC) approach $[9,10]$, where shallow-depth circuits are used at the expense of increased sampling demand. The prominent example of the HQC protocol is the variational quantum eigensolver (VQE) [11-14] that triggered the search for a wide range of variational quantum algorithms. HQC approaches are considered as a viable near-term strategy in quantum computing for chemistry $[15,16]$, optimization [17-19], and quantum machine learning [20]. However, while operating at the reduced circuit depth, variational quantum algorithms also bear significant challenges, including: (1) vanishing gradients when optimizing deep quantum circuits [21-23]; (2) the need for a suitable ansatz capable of preparing a solution state [24]; and (3) a drastic increase in the number of terms to measure when considering the Hamiltonian averaging technique [25]. Numerous recent improvements include adaptive strategies for the ansatz search [26,27] and automated ansatz optimization [28-30], symmetry-preserving VQE [31,32], natural gradient optimization [33-35], measurement frugal VQE [36-45], quantum subspace search [46], layerwise learning [47], and many others. Variational protocols 
have also been applied to simulate the dynamics, showing promise for strongly correlated systems [48-52]. However, the general limitation of variational approaches also calls for alternative solutions for systems at increasing size.

A different strategy to the state preparation relies on applying a nonunitary operation to effectively cool down the higher-energy states. This can be done by representing them as a linear combination of unitaries (LCU) [53]. Being a valuable technique used in large-depth protocols for state-of-the-art Hamiltonian dynamics simulation [54] and linear systems of equations [55], the value of LCU approaches was also assessed for near-term devices and analog simulators where unitary evolution is an available resource. In this way by measuring wavefunction overlaps one can perform inverse quantum iteration [56], quantum Krylov iteration [57], and quantum filter diagonalization [58] to study low-energy properties of correlated materials and molecules. This approach was connected to the quantum version of time grid methods that were used for the Schrödinger equation in the past [59]. From the variational protocols perspective, the LCU ansatz was also applied to chemistry [60] and linear algebra problems [61], notably showing the ability to avoid vanishing gradient regions.

While the requirements for the circuit depth and qubit overhead necessary to perform the SWAP test [62] increase overall, the quantum time grid methods provide a way to reach the global energy minimum when starting from a suitable initial state. As these approaches rely on the simulation of unitary dynamics for the system, they can be seen as relatives of the quantum phase estimation algorithm (QPEA) [63-66]. However, quantum time grid methods use the classical postprocessing of overlaps and unlike QPEA do not require implementation of the controlled unitary dynamics. This makes them suitable for noisy large-scale quantum (NLSQ) devices, where doubling the system size is a less pertinent problem than challenges of the variational search. Time grid methods are particularly favorable for analog quantum simulators $[67,68]$ where one has access to the simulation of quantum dynamics and the overlap measurement $[69,70]$. Importantly, in this case variational approaches and Hamiltonian averaging are not applicable, and quantum time grid approaches offer the way to study low-energy physics.

In this paper we propose a method to approximate a Hamiltonian operator with a sparse sum of unitary propagators. This serves as a building block for many LCU protocols, and is required when measuring the energy of the system in variational approaches, performing direct Hamiltonian iteration [71], implementing Lanczos algorithms, measuring the density of states [72], etc. We use a distinct differential decomposition approach, where Hamiltonian action is simulated with term-by-term quantum evolution, and the resulting energy expectation is read out as a sum of normalized overlaps. This strategy favors analog quantum simulation devices, where energy measurement through string-based Hamiltonian averaging is typically inaccessible. Comparing the proposed approach to the variational quantum eigensolver for the spin-1/2 Heisenberg model at increasing system sizes, we find that the Hamiltonian operator approximation combined with quantum filter diagonalization offers better performance for lattices with more than 12 qubits, suggesting that the strategy can be used for ground-state search in cases where variational methods suffer from the vanishing gradient problem.

\section{METHODOLOGY}

Our goal is to represent a Hamiltonian operator $\hat{\mathcal{H}}$ as a sum of unitary operators, taking as few terms as possible. Typically, this is done by partitioning the Hamiltonian into groups of qubit string operators (mutually commuting inside each group), as described in the Hamiltonian averaging technique used to estimate the energy of the system $[14,73]$. However, certain Hamiltonians, for instance in quantum chemistry, have unfavorable scaling for the number of groups (partitions) to be measured, as it grows like $O\left(N^{4}\right)$ ( $N$ is a number of qubits) for vanilla Hamiltonian averaging [25]. Instead, we use the differential representation based on the quantum evolution operator (propagator) denoted as $\hat{\mathcal{U}}(t)=\exp (-i \hat{\mathcal{H}} t)$, where $t$ is a parameter that defines the evolution time, $\hat{\mathcal{H}}$ is a Hermitian time-independent operator, and we use $\hbar=1$ throughout the paper. Acting with the time derivative operator on the propagator, we can formally write $d \hat{\mathcal{U}} / d t=(-i \hat{\mathcal{H}}) \hat{\mathcal{U}}(t)$. The Hamiltonian operator then reads

$$
\hat{\mathcal{H}}=\left.i \hat{\mathcal{U}}^{\dagger}\left(t_{0}\right) \frac{d}{d t} \hat{\mathcal{U}}(t)\right|_{t \rightarrow t_{0}},
$$

defined using its derivative at some fixed point of time $t_{0}$, and it is convenient to use $t_{0}=0$. We approximate the propagator derivative using the finite difference scheme with $S$ stencil points. The accuracy of the approximation scales as $O\left(\delta t^{S-1}\right)$ [74], where $\delta t$ is the distance between neighboring points. The derivative is approximated as a sum of unitary operators

$$
\left.\frac{d \hat{\mathcal{U}}(t)}{d t}\right|_{t \rightarrow t_{0}}=\frac{1}{\delta t} \sum_{n=-s}^{S-s-1} q_{n}(s) \hat{\mathcal{U}}\left(t_{0}+n \delta t\right)+O\left(\delta t^{S-1}\right)
$$

where $s$ is a shifting parameter that is arbitrary in general, and we usually choose it to be $(S-1) / 2$ for symmetry reasons. To find the expansion coefficients $q_{n}(s)$, we decompose our function at stencil points $t_{0}+n \delta t(n=$ $-s,-s+1, \ldots, S-s+1)$ into Taylor series around $t_{0}$, $f\left(t_{0}+n \delta t\right)=\sum_{j=0}^{S-1}\left[(n \delta t)^{j} / j !\right] d^{j} f(t) /\left.d t^{j}\right|_{t \rightarrow t_{0}}$ [75]. Forming equations for each stencil point, we keep only the first $S$ terms in the expansions. Next, we need to compose a linear combination of these equations such that the coefficient 
before the first derivative is equal to 1 , and coefficients before all the other terms on the right are equal to zero. For this, we solve the eigenvalue equation $\mathbf{M} \cdot \mathbf{q}=\boldsymbol{\delta}$, where the matrix $\mathbf{M}$ reads

$$
\mathbf{M}=\left(\begin{array}{cccc}
1 & 1 & \cdots & 1 \\
-s & -s+1 & \cdots & S-s-1 \\
(-s)^{2} & (-s+1)^{2} & \cdots & (S-s-1)^{2} \\
& & \cdots & \\
(-s)^{S-1} & (-s+1)^{S-1} & \cdots & (S-s-1)^{S-1}
\end{array}\right)
$$

$\mathbf{q}=\left(q_{-s}, q_{-s+1}, \ldots, q_{S-s-1}\right)^{\top}$ is a vector of coefficients we want to find, and we set $\delta=(0,1, \ldots, 0)^{\top}$ (as the first derivative term is considered). By inverting the matrix we find the required coefficients $q_{n}(s)$ and by inserting Eq. (2) into Eq. (1) we write the $S$-stencil differential approximation for the operator $\hat{\mathcal{H}}$ as

$$
\hat{\mathcal{H}} \approx \frac{i}{\delta t} \sum_{n=-s}^{S-s-1} q_{n}(s) e^{-i \hat{\mathcal{H}}(n \delta t)}=: \hat{H}_{\mathrm{diff}}(S, \delta t) .
$$

We refer to this procedure as the Hamiltonian operator approximation (HOA).

\section{RESULTS}

We proceed simulating complex quantum systems and study their low-energy properties using the proposed Hamiltonian operator approximation approach. As test systems we choose several paradigmatic material science models. These include the Heisenberg model of spin-1/2 particles in the external magnetic field, and the strongly correlated hydrogen chain as a standard example from quantum chemistry. For the simulation, we choose the programming language Julia and use the Yao.jl package [76] as a simulation backend, capable of performing quantum protocols with state-of-the-art efficiency [76-78].

\section{A. Energy estimation}

As a useful application for the $\hat{\mathcal{H}}$ dynamical approximation, we consider the measurement of the expected energy. Usually, this is done through the procedure of Hamiltonian averaging, as commonly used in VQE [14]. We propose to use a hybrid quantum-classical approach, where energy is measured as a combination of wavefunction overlaps. Using HOA [Eq. (4)], we can write the Hamiltonian expectation as $\langle\hat{\mathcal{H}}\rangle \approx(i / \delta t) \sum_{n=-s}^{S-s-1} q_{n}(s)\langle\psi \mid \psi(n \delta t)\rangle$, where energy estimation requires calculating the overlap between the measured state $|\psi\rangle$ and the evolved state $|\psi(n \delta t)\rangle=e^{-i \hat{\mathcal{H}}(n \delta t)}|\psi\rangle$. Importantly, since we use the approximated derivative of the evolution operator, the HOA approach favors short time evolution (see the discussion below).
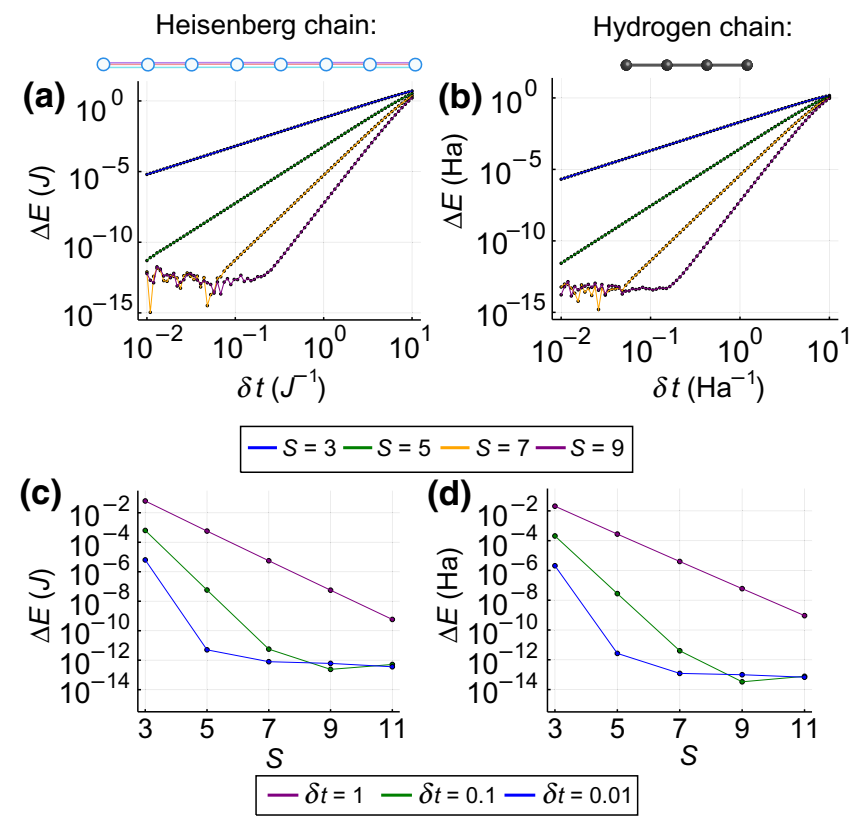

FIG. 1. Energy difference between the exact Hamiltonian expectation and approximate energy $\Delta E$, shown for different systems pictorially presented at the top. The full statevector simulation is performed. (a) Energy difference $\Delta E$ as a function of the time step at different stencil expansions $(S=3,5,7,9)$. We consider the $N=8$ Heisenberg model Hamiltonian with $h / J=0.1$, a uniform state for the measurement, and plot energy in units of the Heisenberg coupling $J$. (b) Energy difference $\Delta E$ as a function of $\delta t$ for several stencil expansions. The Hamiltonian corresponds to the $\mathrm{H}_{4}$ hydrogen chain $(N=8$ qubits) at $d=1.0 \AA$, measured for the Hartee-Fock state. Energy is plotted in Hartree units. (c) Energy difference as a function of the stencil point number $S$, plotted for $\delta t J=1.0,0.1,0.01$. The Hamiltonian is the same as in (a). (d) The energy difference $\Delta E$ shown for different stencil point expansions. The Hamiltonian is the same as in (b).

First, we consider the Heisenberg Hamiltonian of an $N$ qubit chain with open boundaries, $\hat{\mathcal{H}}=-J \sum_{j=1}^{N-1}\left(\hat{X}_{j} \hat{X}_{j+1}\right.$ $\left.+\hat{Y}_{j} \hat{Y}_{j+1}+\hat{Z}_{j} \hat{Z}_{j+1}\right)-h \sum_{j=1}^{N} \hat{Z}_{j}$, where $\hat{X}_{j}, \hat{Y}_{j}, \hat{Z}_{j}$ are Pauli operators acting at site $j$. The results of the simulation are shown in Figs. 1(a) and 1(c) for $N=8$ and $h / J=0.1$. We consider a uniform quantum state created by the string of Hadamard operators as $|\psi\rangle=(|0\rangle+$ $|1\rangle)^{\otimes N} / 2^{N / 2}$, and measure the energy expectation by evaluating terms using noiseless statevector simulation and analog unitary evolution. In Fig. 1(a) we plot the difference between the true expected energy and HOA expectation $\Delta E$ as a function of $\delta t$, plotted for an increasing number of stencil points. We observe that the difference decreases as $O\left(\delta t^{S-1}\right)$, until it reaches the numerical precision-impacted region (see the full discussion on the error scaling in Appendix A). In Fig. 1(c) we plot the energy difference for varying $S$ and observe an exponential improvement in the energy difference, which also requires smaller $\delta t$ (and a correspondingly smaller circuit depth). We note that this 
monotonous dependence holds for the infinite number of measurement shots; the finite shots case is discussed later.

The number of required overlaps to estimate remains small even for increasing $S$, and the complexity of HOA is defined by the maximal propagation phase $T=S \delta t$. While an analog simulation of dynamics highly benefits the proposed approach by construction, we can also use digital simulation of dynamics. Asymptotically optimal simulation of quantum dynamics can be achieved with qubitization [79] or LCU-based approaches [54], at the expense of increased system size due to the ancillary register. A resource-frugal alternative for many near- and midterm applications can be the Trotterization approach, which was previously used for quantum time grid methods $[56,58]$. Recent advances in Trotterized simulation of quantum dynamics suggest that scaling can be improved to $O\left(N T / r+N T^{3} / r^{2}\right)$ for $r$ steps [80], and for local Hamiltonians, there is strong evidence that circuits can be further optimized [81,82]. For the Heisenberg model, Trotterization can be readily performed by alternating couplings on even and odd sublattices [83,84], and implemented in various hardware platforms.

To understand the scaling of HOA with Trotterized quantum evolution, we study the contribution of errors coming from the product formula truncation and differentiation (see Appendix B for the full discussion). As HOA favors a small propagation time to reduce differentiation error, we observe that the number of required Trotter steps remains small for increasing system size. For the $N=13$ spin- $1 / 2$ Heisenberg model, $\delta t J=0.1$, and $S=25$, this allows for a negligible Trotterization error already at $r=5$ steps. The gate count scales as $23 N r+3 N$ and gives 1534 gates. For a hundred-qubit system with similar settings, we get approximately $10^{4}$ gates, which may become possible for improved NLSQ devices.

Next, we proceed to studying quantum chemistry problems, and consider molecular hydrogen (open) chains $\mathrm{H}_{n}$ with homogeneous bond distance $d$ between $n$ atoms. Specifically, we use examples from QunaSys competition obtained using the STO-3G minimal basis set in the spinful case [85]. Qubit-encoded Hamiltonians are then obtained using the Jordan-Wigner transformation from OpenFermion [86]. The chain of four hydrogen atoms $\mathrm{H}_{4}$ at $d=1.0 \AA$ bond distance is encoded using an eight-qubit Hamiltonian. We evaluate the energy expectation of the Hartree-Fock state, providing benchmarking in Figs. 1(b) and $1(\mathrm{~d})$. We find that the overall results are similar to the Heisenberg case, suggesting that the internal structure of the Hamiltonian does not have a major impact on the HOA accuracy.

\section{B. Measurement and noise}

To assess the full performance of the Hamiltonian operator approximation, we account for other imprecision sources coming from the measurement (finite number of shots) and effects of the environment (qubit decay).

The overlap measurement can be performed using several methods. The most popular choices correspond to SWAP and Hadamard tests $[62,87]$. The SWAP test for the $N$-qubit system requires an additional $N+1$ qubits, where one qubit is used for the readout [60]. A destructive version of the SWAP test can be performed using $2 N$ qubits $[69,87]$. The Hadamard test uses a single ancilla added to the register, but doubles the circuit depth $[58,87]$. We also note that in certain cases direct ancilla-free measurement is possible, for instance when circuits possess a specific structure $[88,89]$ or one of the eigenstates is known [56]. Further advances in the area include overlap estimation through randomized measurements [90], atom interferometry [70], or recent projected kernel techniques [91] and shadow tomography [92]. The latter was proven to be optimal for predicting fidelities and entanglement entropy. We consider the improvement in the overlap measurement as an important direction for advancing Hamiltonian approximation techniques.

We simulate the full measurement scheme using the SWAP test to estimate the overlap between the initial state $|\psi\rangle$ and the propagated state. Following the steps in Ref. [60] we prepare a superposition $(1 / \sqrt{2})(|\psi\rangle|0\rangle+$ $i \hat{\mathcal{U}}|\psi\rangle|1\rangle)$ for the system register and an ancilla. We apply the Hadamard gate to the ancilla (last qubit), and measure the expectation for $\hat{Z}_{N+1}$, getting $\left\langle\hat{Z}_{N+1}\right\rangle=$ $-\operatorname{Im}\langle\psi|\hat{\mathcal{U}}| \psi\rangle$. The results are shown in Fig. 2 for the
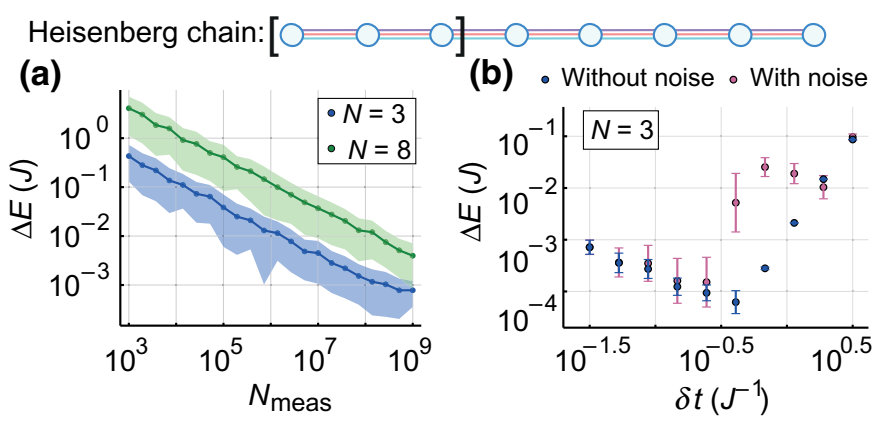

FIG. 2. Influence of the number of measurements on the energy estimate via the SWAP test. (a) Energy difference $\Delta E$ (in units of $J$ ) as a function of the number of measurements for three- and eight-qubit Heisenberg models (bottom and top lines, respectively). We set parameters to $h / J=0.1, S=5$, and $\delta t J=0.5$. To estimate the standard deviation, each simulation is repeated 150 times. (b) Mean energy difference as a function of $\delta t J$ for the Heisenberg model estimated by taking $N_{\text {meas }}=10^{9}$ measurements. Blue dots correspond to the noiseless simulation, with error bars showing the standard deviation. Magenta dots and error bars are for the noisy simulation performed with the wavefunction Monte Carlo approach for $\gamma / J=0.1$. Other parameters are the same as in (a). To estimate the standard deviation, each simulation is repeated 50 times. 
case of the Heisenberg model with $h / J=0.1, S=5$, and $\delta t J=0.5$. We plot the difference between the true energy of the uniform state in the system with Hamiltonian $\hat{\mathcal{H}}$ and the energy of the uniform state calculated with HOA, $\Delta E$, shown as a function of the number of measurement shots $N_{\text {meas }}$ for which $\left\langle\hat{Z}_{N+1}\right\rangle$ is averaged. We observe that the upper bound of the error decreases approximately as $N_{\text {meas }}^{-1 / 2}$ [Fig. 2(a)]. Fixing $N_{\text {meas }}=10^{9}$ we calculate the dependence of the error on the time step $\delta t$. In the absence of noise [blue dots in Fig. 2(b)] the results are in line with the variance scaling $\propto c / N_{\text {meas }}$ ( $c$ being a system-dependent constant) found in Ref. [60]. For relatively large $\delta t$, the error behaves similarly to the ideal statevector simulator; the sampling noise increases the error at $\delta t J<0.3$, meaning that optimal $\delta t$ depends on $N_{\text {meas }}$. This suggests that resolving a small difference between two states (initial and propagated) becomes difficult at very small $\delta t$.

Next, we simulate the effect of noise using the wavefunction Monte Carlo approach [93]. For this, we run multiple trajectories of unitary evolution, subject to probabilistic action of jump operators $\sqrt{\gamma}\left(\hat{X}_{j}+i \hat{Y}_{j}\right) / 2$ that can de-excite each qubit with decay rate $\gamma$. We simulate the Heisenberg model example and plot the energy deviation as a function of $\delta t$ [Fig. 2(b), magenta bars]. The effect of decay is pronounced at large times $\delta t J>1$, where a significant number of jumps leads to erroneous overlap estimates at impacted trajectories (we consider large decay rate $\gamma / J=0.1$, and simulate $10^{9}$ trajectories). As $\delta t$ and the maximal propagation time $T$ decrease, so does the jump probability, leading to improved energy precision $\Delta E$. However, for small $\delta t J<0.1$, we find that even a small number of jumps increases the estimate imprecision, given that each collapsed trajectory leading to the corrupted overlap estimate enters with $\delta t^{-1}$ weight. We observe that optimal $\delta t \approx 0.2 J^{-1}$ lies close to the $N_{\text {meas }}{ }^{-}$ defined case, depending on the decay rate $\gamma$, and we note that a small time step region is the overall preferred choice for HOA.

\section{Direct Hamiltonian iteration}

We generalize our consideration to approximate higher powers of the Hamiltonian operator $\hat{\mathcal{H}}^{k}$. This can be defined through the $k$ th-order derivative of the propagator, $d^{k} \hat{\mathcal{U}} / d t^{k}=(-i \hat{\mathcal{H}})^{k} \hat{\mathcal{U}}(t)$, and following the numerical differentiation at $S$ stencil points we express it as

$$
\hat{\mathcal{H}}^{k}=\frac{1}{(-i \delta t)^{k}} \sum_{n=-S}^{S-s-1} q_{n}^{(k)}(s) e^{-i \hat{\mathcal{H}}(n \delta t)}+O\left(\delta t^{S-k}\right) .
$$

The first nontrivial and useful example corresponds to the approximation of the $\hat{\mathcal{H}}^{2}$ operator, allowing for the straightforward measurement of the variance. This is often required for variational schemes, as together with the energy it may help to detect the convergence of the algorithm [94]. Hamiltonian averaging through the Pauli string measurement has a daunting scaling in this case. We note that, to get the same order of accuracy as for the expansion of $\hat{\mathcal{H}}$, we just need to increase the number of stencil points by one. This holds when going beyond $k=2$, where each power increment requires at least one additional stencil point, and we generally prefer using an odd number of stencil points for the interval to be symmetric. However, the scaling becomes unfavorable for small $\delta t$, with the error growing as $O\left(\delta t^{S-1}\right)$, thus shifting the optimal $\delta t$ region.

We proceed to using HOA as part of the direct iteration algorithm to prepare the dominant eigenstate of the Hamiltonian matrix. We start from an initial state $\left|\Psi_{0}\right\rangle$ having a nonvanishing overlap with the ground state. We simulate the repeated application of $\hat{\mathcal{H}}$ such that at iteration step $k$ we get $\left|\Psi_{k}\right\rangle=\hat{\mathcal{H}}\left|\Psi_{k-1}\right\rangle / \| \hat{\mathcal{H}}\left|\Psi_{k-1}\right\rangle \|$, where the denominator accounts for the state normalization. At sufficiently large $k \mapsto K$ we get $\left|\Psi_{K}\right\rangle=\hat{\mathcal{H}}^{K}\left|\Psi_{0}\right\rangle / \| \hat{\mathcal{H}}^{K}\left|\Psi_{0}\right\rangle \| \approx$ |ground state $\rangle$, and the corresponding expected energy

$$
E_{\text {ground }} \approx\left\langle\Psi_{K}|\hat{\mathcal{H}}| \Psi_{K}\right\rangle=\frac{\left\langle\Psi_{0}\left|\hat{\mathcal{H}}^{2 K+1}\right| \Psi_{0}\right\rangle}{\left\langle\Psi_{0}\left|\hat{\mathcal{H}}^{2 K}\right| \Psi_{0}\right\rangle}
$$

approaches the ground-state energy. We apply the described procedure to prepare a low-energy state for the Heisenberg model at the critical point $h / J=1$ (Fig. 3). Starting from the product state corresponding to a meanfield solution, we lower the energy by one order of magnitude using just four iterations. We also observe that, when $h / J>1$, the convergence further improves. At the same time, we note that direct iteration is typically an unstable procedure with critical dependence on the condition number, and works best for diagonally dominant matrices.

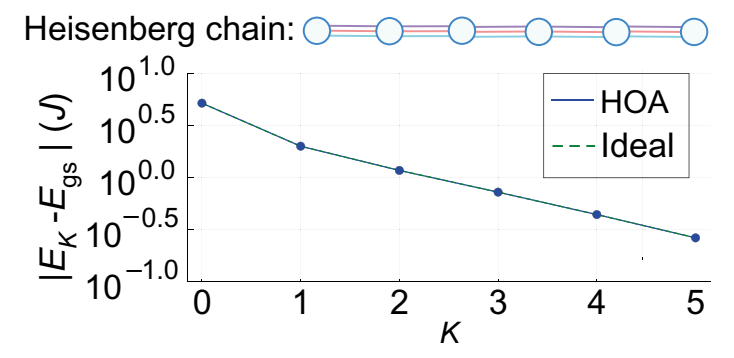

FIG. 3. Difference between the true ground-state energy and the energy from the direct iteration method, where the energy measurement is performed for the ideal and approximated Hamiltonians (lines overlayed). We consider a six-qubit Heisenberg Hamiltonian at the critical point $h / J=1$, and HOA parameters $\delta t=10^{-1.75} \mathrm{~J}^{-1}, S=5$. 


\section{Quantum filter diagonalization with Hamiltonian operator approximation}

Another application where our algorithm provides significant improvement is the quantum filter diagonalization (QFD) proposed by Parrish and McMahon [58]. The goal of the QFD procedure is to find the low-energy spectrum of the system. Importantly, the procedure allows estimating both the ground-state energy and excited state energies using the same resources (overlap measurements), thus being an efficient alternative to constrained variational methods [46,87,95]. In QFD one uses the trial wavefunction as a sum of $2 k_{\max }+1$ propagated states

$$
|\Psi\rangle=\sum_{j} \sum_{k=-k_{\max }}^{k_{\max }} c_{j, k} e^{-i \hat{\mathcal{H}} k / \kappa}\left|\psi_{j}\right\rangle,
$$

where $\left\{\left|\psi_{j}\right\rangle\right\}$ is a set of initial states and $\kappa$ is a spectral width parameter, which is generally greater than the difference between the maximal and minimal eigenvalues $\left|E_{\max }-E_{\min }\right|$. Next, the variational Rayleigh-Ritz approach is applied to find coefficients $c_{j, k}$ such that the energy functional $E=\langle\Psi|\hat{\mathcal{H}}| \Psi\rangle /\langle\Psi \mid \Psi\rangle$ is minimized. This corresponds to solving the generalized eigenvalue problem

$$
\begin{aligned}
& \sum_{j} \sum_{k=-k_{\max }}^{k_{\max }} c_{j, k}\left\langle\psi_{j^{\prime}}\left|e^{i \hat{\mathcal{H}} k^{\prime} / \kappa} \hat{\mathcal{H}} e^{-i \hat{\mathcal{H}} k / \kappa}\right| \psi_{j}\right\rangle \\
& =E \sum_{j} \sum_{k=-k_{\max }}^{k_{\max }} c_{j, k}\left\langle\psi_{j^{\prime}}\left|e^{i \hat{\mathcal{H}} k^{\prime} / \kappa} e^{-i \hat{\mathcal{H}} k / \kappa}\right| \psi_{j}\right\rangle,
\end{aligned}
$$

where overlaps are calculated using a quantum circuit. Substituting $\hat{\mathcal{H}}$ with the differential approximation (4) we avoid the Hamiltonian averaging procedure performed through Pauli string measurements, and the only difference for overlaps in the left- and right-hand sides is the shift in evolution time by $\left(k-k^{\prime}\right) / \kappa$. As setting the time step for QFD requires knowledge of the spectral width $\kappa$, in the following we use the Gershgorin circle theorem to provide its estimate in a scalable way [58].

The results of QFD using the Hamiltonian operator approximation are shown in Fig. 4. First, we consider the $\mathrm{H}_{4}$ hydrogen chain ( $N=8$ qubits) for different bond distances $d=0.5,1.0,2.0 \AA$ and benchmark the difference between the true ground state and the variational state as a function of $k_{\max }$ [Fig. 4(a)]. We observe fast convergence to the true ground state, where starting from the Hartree-Fock initial state we can reach high precision (less than $10^{-5} \mathrm{Ha}$ ) with only a few components in the ansatz. We find that QFD results with the ideal Hamiltonian (dashed curves) and HOA (solid curves) only deviate at larger $k_{\max }$, and strong correlations at larger bond distance lead to a slower convergence rate. As $k_{\max }$ increases,

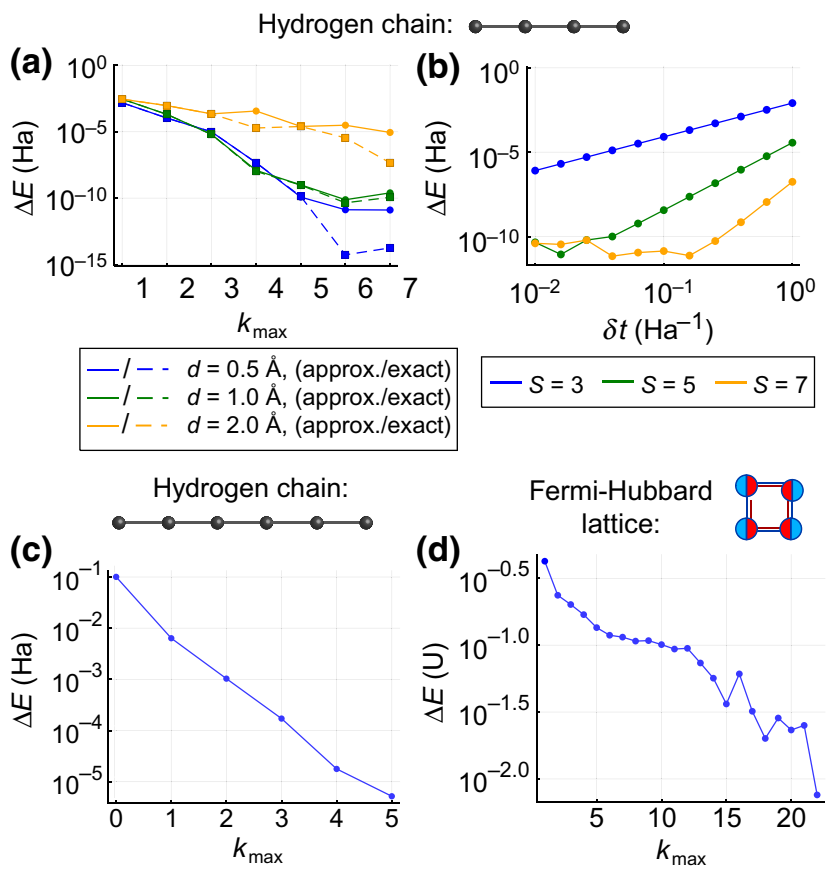

FIG. 4. (a) Energy difference between the lowest state from the quantum filter diagonalization and true ground state, shown as a function of ansatz component number $k_{\max }$. Solid curves correspond to HOA, and dashed curves denote the ideal Hamiltonian expectation. We consider molecular hydrogen $\mathrm{H}_{4}$ at $d=0.5,1.0,2.0 \AA$, and use Gershgorin estimates $\kappa=12.55,5.93,4.19 \mathrm{Ha}$, with $S=5, \delta t=0.01 \mathrm{Ha}^{-1}$. (b) Dependence of the HOA error on $\delta t$ for the $\mathrm{H}_{4}$ string at $d=$ $1.0 \AA, k_{\max }=3, S=5$, and Gershgorin estimate $\kappa=5.93 \mathrm{Ha}$. (c) Energy difference between the QFD+HOA approach and true ground state as a function of $k_{\max }$. We consider the $\mathrm{H}_{6}$ molecule with $d=1.0 \AA, S=5$, and Gershgorin estimate $\kappa=12.86 \mathrm{Ha}$. (d) Energy difference between the QFD+HOA approach and true ground state for the four-site (eight-qubit) two-dimensional Fermi-Hubbard model with $J / U=0.1, \mu / U=0.05, h / U=$ $0.001, \kappa / U=4.60$, uniform initial state, $S=5$ stencils, and $\delta t U=0.01$.

the expressibility of the ansatz improves, both due to a larger number of states and a longer propagation time. However, at large $k_{\max }$ the solution of the generalized eigenvalue problem may be challenging due to instabilities, and care must be taken to choose it in the optimal way. In Fig. 4(b) we confirm that, for fixed QFD parameters, the quality of HOA improves with growing $S$ and decreasing $\delta t$. Going to the larger-scale example of the $N=12$-qubit $\mathrm{H}_{6}$ hydrogen chain $(d=1.0 \AA)$, we again see exponential convergence to the ground-state energy as $k_{\max }$ increases, reaching chemical precision $\Delta E=10^{-3}$ Ha already with five propagated states [Fig. 4(c)].

Finally, we consider the challenging example of a spinful fermion lattice described by the Fermi-Hubbard model. This is described by the Hamiltonian that includes on-site Coulomb repulsion $U$ between opposite spins, 
nearest-neighbor hopping $J$, chemical potential $\mu$ (see the definitions and description in the OpenFermion package [86]). We consider a minimal two-dimensional lattice with four sites, and use the Jordan-Wigner transformation to write the $N=8$ Hamiltonian, where we additionally break the symmetry between up and down spin components with a weak effective magnetic field $h$. Choosing our initial state to be the uniform state, and staying at the half-filling, we observe that the QFD+HOA approach can gradually bring the system towards the low-energy state [Fig. 4(d)]. Importantly, HOA performs so well that no significant difference between the approximation and the ideal Hamiltonian can be spotted, as the two curves overlay. Given the huge progress in analog quantum simulation of Fermi-Hubbard lattices with cold atoms [68] and the possibility to perform interferometric measurements [69], this suggests a promising route towards studying exotic fermion phases.

We note that the problem of preparing the exact ground state of the many-body Hamiltonian is QMA complete [96]. This is defined by the overlap between the initial state and the ground state, which may be exponentially small. However, the same concern holds for quantum phase estimation algorithms. Choosing physically motivated initial states that allow for efficient ground-state preparation is an important goal for future studies in the field.

\section{DISCUSSION}

In this section we discuss the prospects of using the Hamiltonian operator approximation with near- and midterm devices, and compare them to state-of-the-art techniques in the field.

\section{A. VQE comparison}

First, we compare the QFD+HEA approach to the variational quantum eigensolver, being the standard NISQ tool. For this task, we choose the problem of the groundstate energy search for the Heisenberg ring at increasing system size. Specifically, we consider an additional magnetic field, such that the system is at the critical point $(h / J=1)$ with highly entangled eigenstates. For the VQE, we use the state-of-the-art optimization tool set relying on analytic gradients (automatic differentiation) [97,98] and adaptive gradient descent [99]. It is known that automatic differentiation helps to mitigate the noise as compared to numerical differentiation techniques for updating the variational parameters [37]. Specifically, we used the Adam optimizer [99], which is widely used in machine learning and enables the efficient search for optimal variational angles.

We also note that unlike chemistry problems that have a possibility to use the unitary coupled cluster ansatz [15], material science problems typically rely on the hardware efficient strategies, as employed for the Heisenberg model in Refs. $[13,100]$. This corresponds to the ansatz choice as layers of generic rotations (concatenated parameterized rotations $\hat{R}_{Z}-\hat{R}_{X}-\hat{R}_{Z}$ ), followed by the network of controlled NOT (CNOT) gates. Another possibility is to employ alternating operator ansatz, and specifically the Hamiltonian variational ansatz (HVA) with individual tunable blocks based on separate Hamiltonian terms [101]. This can be seen as a physically motivated ansatz for spin systems.

The results for the statevector VQE simulation are shown in Figs. 5(a) and 5(b). We consider Heisenberg rings with the number of spins ranging from 6 to 14, and use the hardware efficient ansatz $[13,100]$. We optimize variational angles for a maximum of 3000 iterations, setting Adam's learning rate to the highest-performing value of 0.001 . We observe that, for smaller system sizes $(N=$ $6,8)$, VQE can prepare the state being close to the true ground state, also staying within a relatively shallow depth [Fig. 5(a)], and a correspondingly small budget [Fig. 5(b)]. As $N$ grows, convergence to the ground state requires an ever increasing depth, and the corresponding gate count
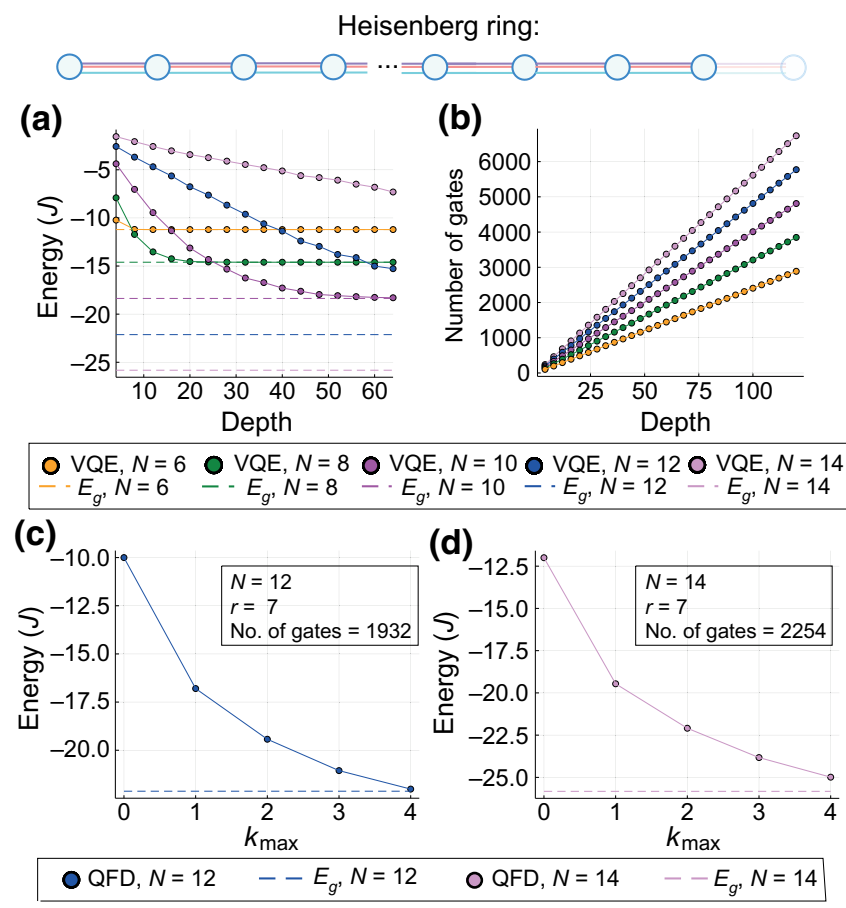

FIG. 5. (a) Ground energy obtained from the VQE approach, shown as a function of the variational circuit depth. We consider the spin-1/2 Heisenberg chain with $6,8,10,12$, and 14 qubits (ring geometry). Results are shown for 3000 iterations of the Adam optimizer, with analytical derivatives and a learning rate of 0.001 . (b) Total number of gates in a single variational circuit shown for increasing circuit depth. (c),(d) Ground-state energy of the Heisenberg ring estimated by the QFD+HOA method. Systems of 12 and 14 qubits are considered as a function of dynamical basis set size $k_{\max }$. We use a second-order Trotterization with $r=7$ Trotter steps. 
for reaching an approximate ground state at $N=14$ surpasses 6000 gates (depth of approximately 100). While we do not yet observe the zero gradients regime, the ground-state preparation requires a largely overparameterized circuit. Previously, VQE with a depth of 73 VQE was simulated for the same Heisenberg model at criticality for $N=7$ qubits [100]. We perform additional tests with HVA (see the details in Appendix C), and observe similar convergence.

Now, let us check the cost of the quantum time grid approach, corresponding to the HOA+VQE approach for the same system. We use the Trotterization approach detailed in Appendix B. We find that, for small systems, the gate count from Trotterization dominates the budget of the QFD+HOA approach, making it more costly than VQE for $N$ smaller than ten qubits. However, at $N=12$ it converges to the ground state with just nine propagated states and below 2000 gates. For $N=14$, the convergence holds, needing 2250 gates for the evolution. The weak scaling suggests that our approach has a larger constant as a starting budget, but outperforms VQE as the system grows to relevant sizes. We also note that our approach does not rely on optimization, and naturally avoids the barren plateaus problem expected as the system scales to a size of approximately 20 qubits [21]. These factors make the proposed approach suitable for NLSQ devices, while the point where it becomes beneficial depends on the task and hardware properties.

Comparing the measurement cost we see that, for increased system sizes, VQE needs over 3000 iterations for convergence. Taking $N=14$ as an example, for each iteration, the gradient measurement requires the number of independent runs to be equal to six times the number of variational parameters (about $6 \times 2000$ ), times the number of measurement shots (needs to be greater than $10^{5}$ for Adam, as shown in Ref. [37]). This leads to a measurement budget of $3 \times 10^{12}$ shots. Our approach requires in general $N_{\text {overlaps }}=\left(4 k_{\max }+1\right) S$ overlaps, being the number of time points of the QFD method multiplied by the number of points in the HOA method. This number decreases further if we adjust the time step of the HOA method to be equal to the time step in the QFD method. In such a case many overlaps become degenerate, and the procedure only needs the calculation of $\left(4 k_{\max }+1\right)+4$ unique overlaps. For $N=14$, we calculate 21 unique overlaps, meaning that, for the same measurement budget, $10^{11}$ shots can be used to reach extra-high precision on each run. Conducting additional studies to compare the Hamiltonian averaging with HOA, we note a similar $\propto 1 / N_{\text {meas }}$ drop in the variance for observables and overlaps, with the latter requiring a constant overhead. The point at which the total measurement budget for HOA becomes favorable then depends on the number of variational parameters and the number of VQE iterations, ultimately defined by the efficiency of the variational workflow.

\section{B. QPEA comparison}

Next, we compare the proposed approach to quantum phase estimation following Ref. [16]. The goal of QPEA is to determine eigenvalues of a unitary operator $\hat{\mathcal{U}}$, when acting on the corresponding eigenstate. While the full QPEA circuit requires an extended ancillary register and quantum Fourier transform [65], the closest QPEA variation to quantum time grid methods is represented by Kitaev's phase estimation $[63,66]$. This requires only a single ancillary qubit and uses the adaptive protocol. However, to estimate the eigenvalue, it uses the controlled unitary operation for the evolution operator, unlike separate overlap measurements. While in general the implementation of controlled unitaries is complex [102], for the simulation of dynamics, we can rewrite it as a simulation of a modified Hamiltonian with the reduced locality. Taking the Heisenberg model as an example, this requires simulation of effective three-body terms involving the ancilla coupled to other spins. Using the Trotterized evolution, we can decompose each Trotter step into single-qubit rotations, Hadamards, phase gates, and CNOT operations. For QPEA, this translates to controlled two-qubit gates and Toffoli gates, adding a significant (about ten) constant overhead, which increases further if restricted connectivity is considered. Also, QPEA is not applicable to ana$\log$ quantum simulators. The relation between approaches thus depends on the platform and available quantum resources.

\section{Scaling}

Finally, we ask the question: can the Hamiltonian operator approximation become a viable strategy for the task of energy estimation in the near- and midterm future where larger systems are available but remain noisy? The issue of performing efficient energy measurement has recently gained attention [25,38,41-44], and the advances are nicely summarized in Table I of Ref. [25]. In particular, considering vanilla Hamiltonian averaging with commuting Pauli heuristics one gets a simple measurement circuit (constant depth of Pauli rotations), but pays for it with $O\left(N^{4}\right)$ scaling for the number of partitions [14] (which we call the depth-frugal method). On the other hand, the methods based on the basis rotation grouping have much better scaling for the number of partitions being $O(N)$, while requiring measurement circuits with a gate count of $N^{2} / 4$ [42] (we say they correspond to partition-frugal methods). Another related partition-frugal technique corresponds to the unitary partitioning approach proposed in Ref. [39]. The Hamiltonian operator approximation thus takes the ultimate position in partition-frugal methods, where the number of independent terms (overlaps) to measure is minimized, at the expense of increased depth if a digital Hamiltonian simulation is used. The practical utility of HOA then depends on the maximal 
propagation time $T$ and the number of stencil points $S$ being used. Numerically, we find that this number does not depend on the system size or has weak, at most $\log (N)$, dependence. The depth of the corresponding measurement circuit increases with $T$ translating to gate count $\widetilde{O}\left(N^{2} T\right)$ considering Trotterization [82]. However, the propagation time $T=S \delta t$ in HOA favors small values, and in many cases few Trotter steps suffice to reduce errors coming from the product formula (Appendix B). Searching for examples where the Hamiltonian operator approximation offers the advantage is an important task for the future research.

\section{CONCLUSIONS}

We have proposed the Hamiltonian operator approximation technique that allows representing a Hamiltonian $\hat{\mathcal{H}}$ as a linear combination of unitary operators. Using numerical differentiation rules, we rewrite $\hat{\mathcal{H}}$ as a sparse sum of quantum propagators, and benchmark it for relevant problems of energy estimation and ground-state preparation. We find that the expected energy of the quantum system can be measured with high precision once we have access to the simulation of its dynamics, as, for instance, available in analog and digital quantum simulators. This holds in the presence of imperfections, including shot noise and decoherence. We also show how the Hamiltonian operator approximation incorporates naturally in quantum Krylovtype approaches, and helps preparing the ground state of the 12-qubit $\mathrm{H}_{6}$ hydrogen chain using the quantum filter diagonalization. Comparing the proposed approach to the variational quantum eigensolver and Hamiltonian averaging, we see that it can become beneficial both in terms of the gatecount and total number of shots for increasing system size. However, the cross-over point depends on the task and quantum platform.

\section{ACKNOWLEDGMENTS}

We thank Annie Paine and Salvatore Chiavazzo for useful discussions on the subject and reading the manuscript. T.A.B. thanks the University of Exeter for hosting her visit during the MSc project work. The authors acknowledge the support from the Mega-Grant No. 14.Y26.31.0015 of the Ministry of Education and Science of the Russian Federation and ITMO Fellowship and Professorship Program.

Note added.-Recently, we became aware of the independent work by Seki and Yunoki [103] that has been carried out in parallel.

\section{APPENDIX A: APPROXIMATION ERRORS}

The Hamiltonian operator approximation relies on differentiation of the evolution operator, and introduces errors that depend on the finite differencing procedure. Our goal is to bound an approximation error as $\left\|\hat{\mathcal{H}}-\hat{H}_{\text {diff }}(S, \delta t)\right\|<\epsilon$, where $\epsilon$ is a predefined constant. This will be achieved for a minimal product of the step size and the total number of points in the differentiation grid, $S \delta t$. Additionally, we note that a small $S$ expansion is generally favored due to its simplicity, and larger $\delta t$ helps at the stage where physical implementation errors are introduced. The approximation error is a function of $S, \delta t$, and the Hamiltonian structure. Using the expansion procedure discussed before, for the infinite-precision arithmetic case, the truncation error reads

$$
\left\|\hat{\mathcal{H}}-\hat{H}_{\text {diff }}(S, \delta t)\right\|<C_{S}(\delta t)^{S} \max _{t}\left\|\frac{d^{S}}{d t^{S}} e^{-i t \hat{\mathcal{H}}}\right\|
$$

where $C_{S}$ is a coefficient depending on the expansion. This suggests that the approximation improves as $S$ increases, and small $\delta t$ is beneficial. Similarly, we can write the bound for an expectation value of the Hamiltonian operator, and introduce a required expected energy precision as $\langle\psi|\hat{\mathcal{H}}| \psi\rangle-\left\langle\psi\left|\hat{H}_{\text {diff }}(S, \delta t)\right| \psi\right\rangle<\epsilon_{\text {energy }}$. We further note that the scaling for HOA is in fact more involved as we approach the limit $\delta t \rightarrow 0$ [74] and finite precision arithmetic is used. In this case the round-off error becomes important, and difference estimation is limited by minimal tolerance $\epsilon_{\text {energy }}^{*} /\|\hat{\mathcal{H}}\|$, typically of the order of $10^{-16}$. For instance, in the case of the quadratic approximation of the derivative the total scaling reads as $\left\|\hat{\mathcal{H}}-\hat{H}_{\text {diff }}(1, \delta t)\right\|<\varepsilon_{\text {appr }}+$ $\varepsilon_{\text {num }}$, where $\varepsilon_{\text {appr }}=\frac{1}{6}(\delta t)^{2} \max _{t}\left\|d^{S}(\exp [-i t \hat{\mathcal{H}}]) / d t^{S}\right\|$ is the approximation error as in Eq. (A1), and $\varepsilon_{\text {num }}=$ $\left(\epsilon^{*} / \delta t\right) \max _{t}\|\exp [-i t \hat{\mathcal{H}}]\|$ comes from the finite precision. We find that at small $\delta t$ the finite-precision rounding error $\varepsilon_{\text {num }}$ starts to dominate, and there exists $\delta t^{*}$ such that the sum of the two contributions is minimized. However, we note that this limit is physically infeasible when the full measurement procedure is considered (see the discussion in the main text).

\section{APPENDIX B: TROTTERIZATION ERRORS}

We aim to understand the scaling of the Hamiltonian operator approximation for systems with a Trotterized simulation of dynamics at increasing system size. For this, we compare errors coming from the differential representation and Trotterization.

Specifically, we choose to simulate quantum dynamics digitally using the second-order Trotterization approach [80], and taking the Heisenberg Hamiltonian as an example. The Hamiltonian evolution for time $\tau$ can be 
simulated as

$$
\begin{aligned}
e^{-i \hat{\mathcal{H}} \tau} \approx( & \prod_{j=N}^{1} e^{(i J \tau / 2 r) \hat{X}_{j} \hat{X}_{j+1}} \prod_{j=N}^{1} e^{(i J \tau / 2 r) \hat{Y}_{j} \hat{Y}_{j+1}} \\
& \times \prod_{j=N}^{1} e^{(i J \tau / 2 r) \hat{Z}_{j} \hat{Z}_{j+1}} \prod_{j=1}^{N} e^{(i h \tau / r) \hat{Z}_{j}} \\
& \times \prod_{j=1}^{N} e^{(i J \tau / 2 r) \hat{Z}_{j} \hat{Z}_{j+1}} \prod_{j=1}^{N} e^{(i J \tau / 2 r) \hat{Y}_{j} \hat{Y}_{j+1}} \\
& \left.\times \prod_{j=1}^{N} e^{(i J \tau / 2 r) \hat{X}_{j} \hat{X}_{j+1}}\right)^{r}
\end{aligned}
$$

where equality is valid for $J \tau \ll 1$ and $r \gg 1$. For the chains with the periodic boundary, we set indices as $N+1=1$. To simulate the circuit, it is convenient to use Mølmer-Sørensen gates $X X_{j, j+1}(\varphi)=\exp \left(-i \varphi \hat{X}_{j} \hat{X}_{j+1}\right)$ [104-106] acting on qubits $j$ and $(j+1)$ for phase $\varphi$. Mølmer-Sørensen gates are native to the trapped ions platform, and can be implemented efficiently beyond nearest-neighbor connectivity. To simulate Heisenberg terms $Y Y_{j, j+1}$, we then conjugate $X X_{j, j+1}$ with the pairs of phase gates $S$ acting at qubits $j$ and $(j+$ 1). Namely, $Y Y_{j, j+1}(\varphi)=S_{j} S_{j+1} X X_{j, j+1}(\varphi) S_{j}^{\dagger} S_{j+1}^{\dagger}$, where $S=\operatorname{diag}(1, i)$. Similarly, we get $Z Z_{j, j+1}$ by conjugating $X X_{j, j+1}$ with Hadamard gates $H$ as $Z Z_{j, j+1}(\varphi)=$ $H_{j} H_{j+1} X X_{j, j+1}(\varphi) H_{j} H_{j+1}$.

In Fig. 6 we show how the error caused by Trotterization [Fig. 6(a)] compares to the overall error of the HOA [Fig. 6(b)]. We see that, as the propagation times for HOA itself are relatively small, only a small number of Trotter steps are needed for the error caused by Trotterization to become much less than the HOA error. For five- and eightqubit Heisenberg rings, the error caused by Trotterization is small already at $r=1$. For a larger system of 13 qubits

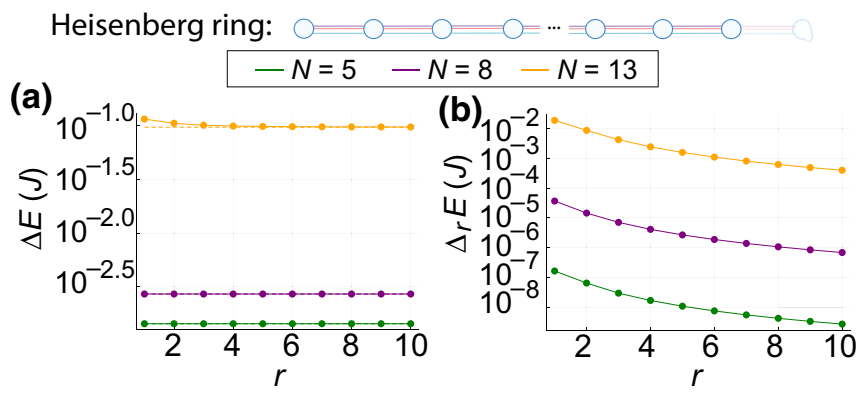

FIG. 6. (a) Difference between the energy obtained with the Trotterized HOA and exact energy of a state in the Heisenberg ring of $N=5,8,13$ qubits. Parameters of the HOA approximation are $S=25, \delta t=0.1$. (b) Difference between the energy obtained with the Trotterized HOA and the energy from HOA with ideal evolution. The parameters are the same as in (a). with the same HOA parameters, it is enough to have five Trotter steps for the Trotter error to become negligible. In the simulation for Fig. 6 we use values $\delta t J=0.1$ and $S=25$, but note that typically these can be smaller, leading to an even more pronounced decrease of the Trotterization error.

When considering the energy measurement as a part of the ground-state preparation process, we see that the maximal evolution time increases. In the QFD+HOA approach this is posed by larger $k_{\max }$ as the system grows. In this case we expect increasing importance of the Trotterization error that translates to larger gatecounts.

\section{APPENDIX C: VQE WITH HAMILTONIAN VARIATIONAL ANSATZ}

In the main text for the VQE comparison we use the hardware-efficient ansatz based on layers of arbitrary single-qubit rotations and CNOT gates. Additionally, we check the performance of the Hamiltonian variational ansatz for improving VQE convergence. The HVA circuit includes layers of Hamiltonian terms corresponding to $\hat{X}_{j} \hat{X}_{j+1}, \hat{Y}_{j} \hat{Y}_{j+1}, \hat{Z}_{j} \hat{Z}_{j+1}$, and $\hat{Z}_{j}$ evolution. Additional $\hat{R}_{X}$ and $\hat{R}_{Z}$ rotations are included to improve expressivity. We used stochastic gradient descent in the Adam form with a learning rate of 0.001 and 3000 iterations to achieve convergence. The results are shown in Fig. 7. We observe the initial decrease in the variational state energy as increasing depth $(d \sim N)$, but note that circuits are not expressive enough to prepare the ground state with high fidelity. After a depth of twenty layers, VQE reaches the barren plateaus, and halts the efficient search. With a further increase in the depth the overparametrization of the ansatz improves the search, reaching a high-quality solution at large depth. We note that small gradients in this regime require an increased number of shots to navigate the derivative-based optimization.

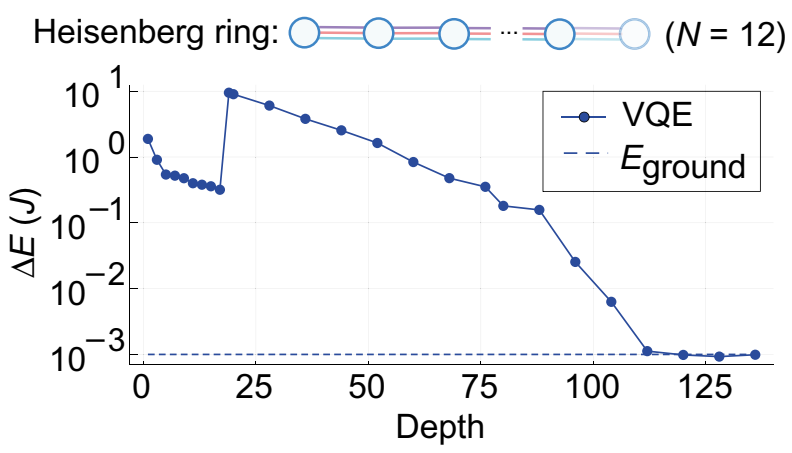

FIG. 7. Energy difference of the final VQE run and the true ground state (log scale), shown as a function of the HVA depth. The problem is the same as in Fig. 5, $N=12$. 
[1] F. Arute et al., Quantum supremacy using a programmable superconducting processor, Nature 574, 505 (2019).

[2] A. Kandala, K. Temme, A. D. Corcoles, A. Mezzacapo, J. M. Chow, and J. M. Gambetta, Error mitigation extends the computational reach of a noisy quantum processor, Nature 567, 491 (2019).

[3] M. Ganzhorn, D. J. Egger, P. Barkoutsos, P. Ollitrault, G. Salis, N. Moll, M. Roth, A. Fuhrer, P. Mueller, S. Woerner, I. Tavernelli, and S. Filipp, Gate-Efficient Simulation of Molecular Eigenstates on a Quantum Computer, Phys. Rev. Appl. 11, 044092 (2019).

[4] Y. Nam et al., Ground-state energy estimation of the water molecule on a trapped ion quantum computer, $\mathrm{Npj}$ Quantum Inf. 6, 33 (2020).

[5] Google AI Quantum and Collaborators, F. Arute, K. Arya, R. Babbush, D. Bacon, J. C. Bardin, R. Barends, S. Boixo, M. Broughton, B. B. Buckley et al., Hartree-Fock on a superconducting qubit quantum computer, Science 369, 1084 (2020)

[6] M. A. Nielsen and I. L. Chuang, Quantum Computation and Quantum Information (Cambridge Univ. Press, New York, USA, 2010).

[7] J. Preskill, Quantum computing in the NISQ era and beyond, Quantum 2, 79 (2018).

[8] K. Bharti, A. Cervera-Lierta, Thi Ha Kyaw, T. Haug, S. Alperin-Lea, Abhinav Anand, M. Degroote, H. Heimonen, J. S. Kottmann, T. Menke, Wai-Keong Mok, Sukin Sim, Leong-Chuan Kwek, and A. Aspuru-Guzik, Noisy intermediate-scale quantum (NISQ) algorithms, arXiv:2101.08448 [quant-ph].

[9] N. Moll, P. Barkoutsos, L. S. Bishop, J. M. Chow, A. Cross, D. J. Egger, S. Filipp, Andreas Fuhrer, J. M. Gambetta, M. Ganzhorn, A. Kandala, A. Mezzacapo, P. Müller, W. Riess, G. Salis, J. Smolin, I. Tavernelli, and K. Temme, Quantum optimization using variational algorithms on near-term quantum devices, Quantum Sci. Technol. 3, 030503 (2018).

[10] M. Cerezo, A. Arrasmith, R. Babbush, S. C. Benjamin, S. Endo, K. Fujii, J. R. McClean, K. Mitarai, Xiao Yuan, L. Cincio, and P. J. Coles, Variational Quantum Algorithms, arXiv:2012.09265 [quant-ph].

[11] A. Peruzzo, J. McClean, P. Shadbolt, M.-H. Yung, X.-Q. Zhou, P. J. Love, A. Aspuru-Guzik, and J. L. O'Brien, A variational eigenvalue solver on a photonic quantum processor, Nat. Comm. 5, 4213 (2014).

[12] P. J. J. O’Malley, R. Babbush, I. D. Kivlichan, J. Romero, J. R. McClean, and R. Barends et al., Scalable Quantum Simulation of Molecular Energies, Phys. Rev. X 6, 031007 (2016).

[13] A. Kandala, A. Mezzacapo, K. Temme, M. Takita, M. Brink, J. M. Chow, and J. M. Gambetta, Hardwareefficient variational quantum eigensolver for small molecules and quantum magnets, Nature (London) 549, 242 (2017).

[14] J. McClean, J. Romero, R. Babbush, and A. AspuruGuzik, The theory of variational hybrid quantum-classical algorithms, New J. Phys. 18, 023023 (2016).

[15] S. McArdle, S. Endo, A. Aspuru-Guzik, S. C. Benjamin, and Xiao Yuan, Quantum computational chemistry, Rev. Mod. Phys. 92, 15003 (2020).
[16] Y. Cao, J. Romero, J. P. Olson, M. Degroote, P. D. Johnson, M. Kieferová, I. D. Kivlichan, T. Menke, B. Peropadre, N. P. D. Sawaya, Sukin Sim, L. Veis, and A. Aspuru-Guzik, Quantum chemistry in the age of Quantum computing, Chem. Rev. 119, 10856 (2019), arXiv:1812.09976.

[17] E. Farhi, J. Goldstone, and S. Gutmann, A Quantum Approximate Optimization Algorithm, arXiv:1411.4028 (2014).

[18] M. P. Harrigan, K. J. Sung, M. Neeley, K. J. Satzinger, F. Arute, K. Arya, J. Atalaya, J. C. Bardin, R. Barends, S. Boixo et al., Quantum approximate optimization of non-planar graph problems on a planar superconducting processor, Nature Physics 17, 332 (2021).

[19] V. Akshay, H. Philathong, M. E. S. Morales, and J. D. Biamonte, Reachability Deficits in Quantum Approximate Optimization, Phys. Rev. Lett. 124, 090504 (2020).

[20] M. Benedetti, E. Lloyd, S. Sack, and M. Fiorentini, Parameterized quantum circuits as machine learning models, Quantum Sci. Technol. 4, 043001 (2019).

[21] J. R. McClean, S. Boixo, Vadim N. Smelyanskiy, Ryan Babbush, and Hartmut Neven, Barren plateaus in quantum neural network training landscapes, Nat. Commun. 9, 4812 (2018)

[22] M. Cerezo, A. Sone, T. Volkoff, L. Cincio, and P. J. Coles, Cost function dependent barren plateaus in shallow parametrized quantum circuits, Nat. Commun. 12, 1791 (2021).

[23] M. Cerezo and P. J. Coles, Higher order derivatives of quantum neural networks with barren plateaus, Quantum Sci. Technol. 6, 035006 (2021).

[24] Y. Herasymenko and T. E. O'Brien, A diagrammatic approach to variational quantum ansatz construction, arXiv:1907.08157 (2019).

[25] X. Bonet-Monroig, R. Babbush, and T. E. O'Brien, Nearly Optimal Measurement Scheduling for Partial Tomography of Quantum States, Phys. Rev. X 10, 031064 (2020).

[26] H. R. Grimsley, S. E. Economou, E. Barnes, and N. J. Mayhall, An adaptive variational algorithm for exact molecular simulations on a quantum computer, Nat. Commun. 10, 3007 (2019).

[27] H. L. Tang, V. O. Shkolnikov, G. S. Barron, H. R. Grimsley, N. J. Mayhall, E. Barnes, and S. E. Economou, QubitADAPT-VQE: An adaptive algorithm for constructing hardware-efficient ansatze on a quantum processor, PRX Quantum 2, 020310 (2021).

[28] M. Ostaszewski, E. Grant, and M. Benedetti, Structure optimization for parameterized quantum circuits, Quantum 5, 391 (2021).

[29] A. G. Rattew, S. Hu, M. Pistoia, R. Chen, and S. Wood, A Domain-agnostic, Noise-resistant, Hardwareefficient Evolutionary Variational Quantum Eigensolver, arXiv:1910.09694 (2019).

[30] D. Chivilikhin, A. Samarin, V. Ulyantsev, I. Iorsh, A. R. Oganov, and O. Kyriienko, MoG-VQE: Multiobjective genetic variational quantum eigensolver, arXiv:2007.04424 (2020).

[31] B. T. Gard, L. Zhu, G. S. Barron, N. J. Mayhall, S. E. Economou, and E. Barnes, Efficient symmetry-preserving state preparation circuits for the variational quantum eigensolver algorithm, Npj Quantum Inf. 6, 10 (2020). 
[32] X. Bonet-Monroig, R. Sagastizabal, M. Singh, and T. E. O'Brien, Low-cost error mitigation by symmetry verification, Phys. Rev. A 98, 062339 (2018).

[33] S. McArdle, T. Jones, S. Endo, Ying Li, S. Benjamin, and Xiao Yuan, Variational ansatz-based quantum simulation of imaginary time evolution, Npj Quantum Inf. 5, 75 (2019).

[34] J. Stokes, J. Izaac, N. Killoran, and G. Carleo, Quantum natural gradient, Quantum 4, 269 (2020).

[35] B. Koczor and S. C. Benjamin, Quantum natural gradient generalised to non-unitary circuits, arXiv:1912.08660 (2019).

[36] A. F. Izmaylov, Tzu-Ching Yen, and I. G. Ryabinkin, Revising measurement process in the variational quantum eigensolver: Is it possible to reduce the number of separately measured operators?, Chem. Sci. 10, 3746 (2019).

[37] J. M. Kübler, A. Arrasmith, L. Cincio, and P. J. Coles, An adaptive optimizer for measurement-frugal variational algorithms, Quantum 4, 263 (2020).

[38] V. Verteletskyi, Tzu-Ching Yen, and A. F. Izmaylov, Measurement optimization in the variational quantum eigensolver using a minimum clique cover, J. Chem. Phys. 152, 124114 (2020).

[39] A. F. Izmaylov, Tzu-Ching Yen, R. A. Lang, and V. Verteletskyi, Unitary partitioning approach to the measurement problem in the variational quantum eigensolver method, J. Chem. Theory Comput. 16, 190 (2020).

[40] Tzu-Ching Yen, V. Verteletskyi, and A. F. Izmaylov, Measuring all compatible operators in one series of single-qubit measurements using unitary transformations, J. Chem. Theory Comput. 16, 2400 (2020).

[41] P. Gokhale, O. Angiuli, Yongshan Ding, Kaiwen Gui, T. Tomesh, M. Suchara, M. Martonosi, and F. T. Chong, Minimizing State Preparations in Variational Quantum Eigensolver by Partitioning into Commuting Families, arXiv:1907.13623 (2019).

[42] W. J. Huggins, J. McClean, N. Rubin, Z. Jiang, N. Wiebe, K. B. Whaley, and R. Babbush, Efficient and noise resilient measurements for quantum chemistry on near-term quantum computers, arXiv:1907.13117 (2019).

[43] O. Crawford, B. van Straaten, D. Wang, T. Parks, E. Campbell, and S. Brierley, Efficient quantum measurement of Pauli operators in the presence of finite sampling error, Quantum 5, 385 (2021).

[44] J. Cotler and F. Wilczek, Quantum Overlapping Tomography, Phys. Rev. Lett. 124, 100401 (2020).

[45] Tzu-Ching Yen and A. F. Izmaylov, Cartan sub-algebra approach to efficient measurements of quantum observables, arXiv:2007.01234 (2020).

[46] K. M. Nakanishi, K. Mitarai, and K. Fujii, Subspacesearch variational quantum eigensolver for excited states, Phys. Rev. Res. 1, 033062 (2019).

[47] A. Skolik, J. R. McClean, M. Mohseni, P. van der Smagt, and M. Leib, Layerwise learning for quantum neural networks, Quantum Mach. Intell. 3, 5 (2021).

[48] Ying Li and S. C. Benjamin, Efficient Variational Quantum Simulator Incorporating Active Error Minimization, Phys. Rev. X 7, 021050 (2017).
[49] Xiao Yuan, Suguru Endo, Qi Zhao, Ying Li, and S. C. Benjamin, Theory of variational quantum simulation, Quantum 3, 191 (2019).

[50] Ming-Cheng Chen, Ming Gong, Xiaosi Xu, Xiao Yuan, Jian-Wen Wang, Can Wang, Chong Ying, Jin Lin, Yu Xu, Yulin $\mathrm{Wu}$, Shiyu Wang, Hui Deng, Futian Liang, ChengZhi Peng, S. C. Benjamin, Xiaobo Zhu, Chao-Yang Lu, and Jian-Wei Pan, Demonstration of Adiabatic Variational Quantum Computing with a Superconducting Quantum Coprocessor, Phys. Rev. Lett. 125, 180501 (2020).

[51] Suguru Endo, Jinzhao Sun, Ying Li, Simon C. Benjamin, and Xiao Yuan, Variational Quantum Simulation of General Processes, Phys. Rev. Lett. 125, 010501 (2020).

[52] Suguru Endo, Zhenyu Cai, S. C. Benjamin, and Xiao Yuan, Hybrid quantum-classical algorithms and quantum error mitigation, J. Phys. Soc. Jpn. 90, 032001 (2021).

[53] Guilu Long and Yang Liu, Duality quantum computing, Front. Comput. Sci. China 2, 167 (2008).

[54] D. W. Berry, A. M. Childs, R. Cleve, R. Kothari, and R. D. Somma, Simulating Hamiltonian Dynamics with a Truncated Taylor Series, Phys. Rev. Lett. 114, 090502 (2015).

[55] A. M. Childs, R. Kothari, and R. D. Somma, Quantum algorithm for systems of linear equations with exponentially improved dependence on precision, SIAM J. Comput. 46, 1920 (2017).

[56] O. Kyriienko, Quantum inverse iteration algorithm for programmable quantum simulators, Npj Quantum Inf. 6, 7 (2020).

[57] N. H. Stair, R. Huang, and F. A. Evangelista, A multireference quantum Krylov algorithm for strongly correlated electrons, J. Chem. Theory Comput. 16, 2236 (2020).

[58] R. M. Parrish and P. L. McMahon, Quantum Filter Diagonalization: Quantum Eigendecomposition without Full Quantum Phase Estimation, arXiv:1909.08925 (2019).

[59] G. G. Balint-Kurti, R. N. Dixon, and C. C. Marston, Grid methods for solving the Schrödinger equation and time dependent quantum dynamics of molecular photofragmentation and reactive scattering processes, Int. Rev. Phys. Chem. 11, 317 (1992).

[60] W. J. Huggins, J. Lee, U. Baek, B. O'Gorman, and K. B. Whaley, A non-orthogonal variational quantum eigensolver, New J. Phys. 22, 073009 (2020).

[61] Hsin-Yuan Huang, K. Bharti, and P. Rebentrost, Nearterm quantum algorithms for linear systems of equations, arXiv:1909.07344 (2019).

[62] A. K. Ekert, C. M. Alves, D. K. L. Oi, M. Horodecki, P. Horodecki, and L. C. Kwek, Direct Estimations of Linear and Nonlinear Functionals of a Quantum State, Phys. Rev. Lett. 88, 217901 (2002).

[63] A. Yu. Kitaev, Quantum measurements and the abelian stabilizer problem, arXiv:quant-ph/9511026 (1996).

[64] D. S. Abrams and S. Lloyd, Quantum Algorithm Providing Exponential Speed Increase for Finding Eigenvalues and Eigenvectors, Phys. Rev. Lett. 83, 5162 (1999).

[65] A. Aspuru-Guzik, A. D. Dutoi, P. J. Love, and M. HeadGordon, Simulated quantum computation of molecular energies, Science 309, 1704 (2005). 
[66] M. Dobsicek, G. Johansson, V. Shumeiko, and G. Wendin, Arbitrary accuracy iterative quantum phase estimation algorithm using a single ancillary qubit: A two-qubit benchmark, Phys. Rev. A 76, 030306(R) (2007).

[67] I. Bloch, J. Dalibard, and S. Nascimbéne, Quantum simulations with ultracold quantum gases, Nat. Phys. 8, 267 (2012).

[68] A. Mazurenko, C. S. Chiu, G. Ji, M. F. Parsons, M. Kanász-Nagy, R. Schmidt, F. Grusdt, E. Demler, D. Greif, and M. Greiner, A cold-atom fermi-hubbard antiferromagnet, Nature 545, 462 (2017).

[69] R. Islam, Ruichao Ma, P. M. Preiss, M. E. Tai, A. Lukin, M. Rispoli, and M. Greiner, Measuring entanglement entropy in a quantum many-body system, Nature (London) 528, 77 (2015).

[70] J. Cotler, Soonwon Choi, A. Lukin, H. Gharibyan, T. Grover, M. E. Tai, M. Rispoli, R. Schittko, P. M. Preiss, A. M. Kaufman, M. Greiner, H. Pichler, and P. Hayden, Quantum Virtual Cooling, Phys. Rev. X 9, 031013 (2019).

[71] Yimin Ge, J. Tura, and J. I. Cirac, Faster ground state preparation and high-precision ground energy estimation with fewer qubits, J. Math. Phys. 60, 022202 (2019).

[72] A. Weiße, G. Wellein, A. Alvermann, and H. Fehske, The kernel polynomial method, Rev. Mod. Phys. 78, 275 (2006).

[73] J. R. McClean, R. Babbush, P. J. Love, and A. AspuruGuzik, Exploiting locality in quantum computation for quantum chemistry, J. Phys. Chem. Lett. 5, 4368 (2014).

[74] Knut Mørken, Numerical Algorithms and Digital Representation (lecture course, Univ. Oslo, Norway, 2010), Chap. 11.

[75] B. Fornberg, Generation of finite difference formulas on arbitrarily spaced grids, Math. Comp. 51, 699 (1988).

[76] Xiu-Zhe Luo, Jin-Guo Liu, Pan Zhang, and Lei Wang, Yao.jl: Extensible, efficient framework for quantum algorithm design, Quantum 4, 341 (2020).

[77] Jinfeng Zeng, Yufeng Wu, Jin-Guo Liu, Lei Wang, and Jiangping $\mathrm{Hu}$, Learning and inference on generative adversarial quantum circuits, Phys. Rev. A 99, 052306 (2019).

[78] Jin-Guo Liu, Yi-Hong Zhang, Yuan Wan, and Lei Wang, Variational quantum eigensolver with fewer qubits, Phys. Rev. Res. 1, 023025 (2019).

[79] Guang Hao Low and I. L. Chuang, Hamiltonian simulation by qubitization, Quantum 3, 163 (2019).

[80] Minh C. Tran, Su-Kuan Chu, Yuan Su, A. M. Childs, and A. V. Gorshkov, Destructive Error Interference in ProductFormula Lattice Simulation, Phys. Rev. Lett. 124, 220502 (2020).

[81] A. Childs, D. Maslov, Yunseong Nam, N. J. Ross, and Yuan $\mathrm{Su}$, Toward the first quantum simulation with quantum speedup, PNAS 115, 9456 (2018).

[82] A. M. Childs, Yuan Su, Minh C. Tran, N. Wiebe, and Shuchen Zhu, Theory of Trotter Error with Commutator Scaling, Phys. Rev. X 11, 011020 (2021).

[83] U. L. Heras, A. Mezzacapo, L. Lamata, S. Filipp, A. Wallraff, and E. Solano, Digital Quantum Simulation of Spin Systems in Superconducting Circuits, Phys. Rev. Lett. 112, 200501 (2014).

[84] O. Kyriienko and A. S. Sørensen, Floquet Quantum Simulation with Superconducting Qubits, Phys. Rev. Appl. 9, 064029 (2018).
[85] See online at https:/quantaggle.com/competitions/gs-pes/ and https://github.com/qulacs/Quantaggle_dataset/tree/ master/datasets/Small_Molecules_1.

[86] J. R. McClean et al., OpenFermion: The electronic structure package for quantum computers, Quantum Sci. Technol. 5, 034014 (2020).

[87] O. Higgott, Daochen Wang, and S. Brierley, Variational quantum computation of excited states, Quantum 3, 156 (2019).

[88] K. Mitarai and K. Fujii, Methodology for replacing indirect measurements with direct measurements, Phys. Rev. Res. 1, 013006 (2019).

[89] V. Havliček, A. D. Corcoles, K. Temme, A. W. Harrow, A. Kandala, J. M. Chow, and J. M. Gambetta, Supervised learning with quantum-enhanced feature spaces, Nature 567, 209 (2019).

[90] A. Elben, B. Vermersch, R. van Bijnen, C. Kokail, T. Brydges, C. Maier, M. K. Joshi, R. Blatt, C. F. Roos, and P. Zoller, Cross-Platform Verification of Intermediate Scale Quantum Devices, Phys. Rev. Lett. 124, 010504 (2020).

[91] Hsin-Yuan Huang, M. Broughton, M. Mohseni, R. Babbush, S. Boixo, H. Neven, and J. R. McClean, Power of data in quantum machine learning, Nat. Commun. 12, 2631 (2021).

[92] Hsin-Yuan Huang, R. Kueng, and J. Preskill, Predicting many properties of a quantum system from very few measurements, Nat. Phys. 16, 1050 (2020).

[93] J. Dalibard, Y. Castin, and K. Mølmer, Wave-Function Approach to Dissipative Processes in Quantum Optics, Phys. Rev. Lett. 68, 580 (1992).

[94] C. Kokail, C. Maier, R. van Bijnen, T. Brydges, M. K. Joshi, P. Jurcevic, C. A. Muschik, P. Silvi, R. Blatt, C. F. Roos, and P. Zoller, Self-verifying variational quantum simulation of the lattice schwinger model, Nature 569, 355 (2019).

[95] T. Jones, S. Endo, S. McArdle, Xiao Yuan, and S. C. Benjamin, Variational quantum algorithms for discovering hamiltonian spectra, Phys. Rev. A 99, 062304 (2019).

[96] N. Schuch and F. Verstraete, Computational complexity of interacting electrons and fundamental limitations of density functional theory, Nat. Phys. 5, 732 (2009).

[97] K. Mitarai, M. Negoro, M. Kitagawa, and K. Fujii, Quantum circuit learning, Phys. Rev. A 98, 032309 (2018).

[98] M. Schuld, V. Bergholm, C. Gogolin, J. Izaac, and N. Killoran, Evaluating analytic gradients on quantum hardware, Phys. Rev. A 99, 032331 (2019).

[99] D. P. Kingma and J. L. Ba, Adam: A Method For Stochastic Optimisation, arXiv:1412.6980 (2017).

[100] E. Grant, L. Wossnig, M. Ostaszewski, and M. Benedetti, An initialization strategy for addressing barren plateaus in parametrized quantum circuits, Quantum 3, 214 (2019).

[101] R. Wiersema, Cunlu Zhou, Y. de Sereville, J. Felipe Carrasquilla, Yong Baek Kim, and Henry Yuen, Exploring entanglement and optimization within the hamiltonian variational ansatz, PRX Quantum 1, 020319 (2020).

[102] Xiao-Qi Zhou, T. C. Ralph, P. Kalasuwan, Mian Zhang, A. Peruzzo, B. P. Lanyon, and J. L. O'Brien, Adding control to arbitrary unknown quantum operations, Nat. Commun. 2, 413 (2011). 
[103] K. Seki and S. Yunoki, Quantum power method by a superposition of time-evolved states, PRX Quantum 2, 010333 (2021).

[104] A. Sørensen and K. Mølmer, Quantum Computation with Ions in Thermal Motion, Phys. Rev. Lett. 82, 1971 (1999).
[105] K. Mølmer and A. Sørensen, Multiparticle Entanglement of Hot Trapped Ions, Phys. Rev. Lett. 82, 1835 (1999).

[106] D. Maslov, Basic circuit compilation techniques for an ion-trap quantum machine, New J. Phys. 19, 023035 (2017). 